VOL. 64 (2001) [213-231]

\title{
CONVERGENCE THEOREMS FOR TOPOLOGICAL GROUP VALUED MEASURES ON EFFECT ALGEBRAS
}

\author{
Francisco García Mazarío
}

\begin{abstract}
In this paper we study the validity of several convergence theorems for measures defined on an effect algebra and taking values in a Hausdorff commutative topological group. We establish the Brooks-Jewett theorem and the Nikodým convergence theorem, giving as a corollary a result, due to Aarnes, about the convergence of a sequence of normal linear functionals on a von Neumann algebra. We prove two new convergence theorems concerning completely additive and $\tau$-smooth measures, and we obtain also a convergence theorem for regular measures.
\end{abstract}

\section{INTRODUCTION}

In classical measure theory the so-called convergence theorems play an important role. These theorems (such as Brooks-Jewett, Nikodým convergence, Vitali-Hahn-Saks or Nikodým boundedness theorems) state, roughly speaking, that the setwise limit of a sequence of measures having a certain property (to be $s$-bounded, countably additive or $\lambda$-continuous, for example) also has the same property.

We can say that the "classical" versions of such theorems consist of considering scalar measures defined on a $\sigma$-complete Boolean algebra (see [11, Chapter III]). More general versions for measures with values in Banach spaces or topological groups were known twenty years ago (see [10], [32] or [45]). It is well known that convergence theorems need not hold for non $\sigma$-complete Boolean algebras, but they are true for measures defined on certain classes of Boolean algebras that are not $\sigma$-complete (see [10] or [42]). In 1984, Freniche [18] gave a condition for a Boolean algebra (the subsequential interpolation property) under which they are valid (see also [33]). But the results about this subject are not completely settled, and it is possible to find very recent papers in this direction (see [26] or [47]).

On the other hand, motivated by the mathematical foundations of quantum mechanics, so-called non-commutative measure theory has seen important developments. This theory consists in replacing Boolean algebras by other structures (sometimes called quantum logics) such as orthomodular posets, orthoalgebras or, more recently, D-posets

Received 11th October, 2000

Copyright Clearance Centre, Inc. Serial-fee code: 0004-9727/01 \$A2.00+0.00. 
(introduced by Kôpka and Chovanec in 1994 [30]) or effect algebras (Foulis and Bennet, 1994 [16]). These structures are more general than Boolean algebras and, at the same time, extend the properties of the set of all closed subspaces of a Hilbert space (see Example 2.7 below). We have chosen effect algebras because they are a natural generalisation of Boolean algebras and orthoalgebras. Historically, it may be considered that non-commutative measure theory was born in 1957, with the publication of a theorem of Gleason [19], probably one of most important mathematical theorems of this century. The first result about convergence theorems in this setting is due to Aarnes [1] and dates from 1966. But, in the last ten years, the number of mathematical works related to that theory has greatly expanded. Among such works there are several extensions of classical convergence theorems (see [24], [37] or [38]), including generalisations for topological group valued measures (see [4] or [37]) and several versions for non $\sigma$-complete quantum logics satisfying a condition similar to the subsequential interpolation property defined by Freniche in Boolean algebras (see [3] or [24]).

In this paper, we extend some of these results in two ways. First, we consider measures defined on effect algebras, instead of orthomodular posets or orthoalgebras. Moreover, instead of a "sequence of sequences" method, we apply a previous result about a "net of sequences". Later, as a consequence, we obtain, among other things, a convergence theorem for completely additive measures and another one for $\tau$-smooth measures. These theorems were unknown, even in the classical case. We want to emphasise that completely additive measures are very important in the setting of noncommutative theory; in fact, the statement of the Gleason theorem for non separable Hilbert spaces makes use of such measures (see [6] or [36]). Also $\tau$-smooth measures are important in measure theory. Their relationship with regular measures appears, for example, in the study of topological measures or in the classical Alexandroff decomposition theorem [2] (see also [40]).

The paper is organised as follows: in Section 2 we give the definition and basic properties of effect algebras, making a detailed study of orthogonality, and ending with a collection of interesting examples. Section 3 is devoted to the study of measures defined on effect algebras with values in a topological group. Mainly, we deal with $s$-bounded measures, getting several results, in particular Lemma 3.4, which are very important in the proof of subsequent theorems. Section 4 contains the main results of this paper, namely the Brooks-Jewett theorem, the Nikodým convergence theorem (including a version for completely additive measures), a convergence theorem for $\tau$-smooth measures and another one about regular measures. Finally, in Section 5 we include some remarks about the results obtained in preceding sections and about Nikodým boundedness and Vitali-Hahn-Saks theorems. 


\section{EFFECT ALGEBRAS}

Let $L$ be a set with two special elements 0,1 . Let $\perp$ be a subset of $L \times L$, and let $\oplus: \perp \longrightarrow L$ be a binary operation. We say that the algebraic system $(L, \perp, \oplus, 0,1)$ is an effect algebra if the following axioms hold:

(i) (Commutative Law) If $a, b \in L$ and $a \perp b$, then $b \perp a$ and $a \oplus b=b \oplus a$.

(ii) (Associative Law) If $a, b, c \in L, a \perp b$ and $(a \oplus b) \perp c$, then $b \perp c$; $a \perp(b \oplus c)$ and $(a \oplus b) \oplus c=a \oplus(b \oplus c)$.

(iii) (Orthocomplementation Law) For every $a \in L$ there exists a unique $b \in L$ such that $a \perp b$ and $a \oplus b=1$.

(iv) (Zero-Unit Law) If $a \in L$ and $1 \perp a$, then $a=0$.

Let $L=(L, \perp, \oplus, 0,1)$ be an effect algebra. If $a, b \in L$ and $a \perp b$ we say that $a$ and $b$ are orthogonal. If $a \oplus b=1$ we say that $b$ is the orthocomplement of $a$, and we write $b=a^{\prime}$. Clearly $1^{\prime}=0,\left(a^{\prime}\right)^{\prime}=a, a \perp 0$ and $a \oplus 0=a$ for all $a \in L$. We say that $a \leqslant b$ if there exists $c \in L$ such that $a \perp c$ and $a \oplus c=b$. It is easy to prove that $\leqslant$ is a partial ordering on $L$ (that is, $\leqslant$ is reflexive, antisymmetric and transitive) such that $0 \leqslant a \leqslant 1, a \leqslant b \Longleftrightarrow b^{\prime} \leqslant a^{\prime}$ and $a \leqslant b^{\prime} \Longleftrightarrow a \perp b$ for $a, b \in L$.

If $a \leqslant b$, the element $c \in L$ such that $c \perp a$ and $a \oplus c=b$ is unique, and it satisfies the condition $c=\left(a \oplus b^{\prime}\right)^{\prime}$. It will be denoted by $c=b \ominus a$.

Additional properties of effect algebras can be found in the following lemma. For the proof, we refer to [16].

LEMMA 2.1. Let $L$ be an effect algebra. Then, for all $a, b, c \in L$, we have

(i) If $a \leqslant b$, then $b \ominus a \leqslant b$ and $b \ominus(b \ominus a)=a$.

(ii) If $a \leqslant b \leqslant c$, then $c \ominus b \leqslant c \ominus a$ and $(c \ominus a) \ominus(c \ominus b)=b \ominus a$.

(iii) If $a \leqslant b$, then $b \ominus a=0 \Longleftrightarrow a=b$ and $b \ominus a=b \Longleftrightarrow a=0$.

(iv) If $a \leqslant b \leqslant c^{\prime}$, then $a \oplus c \leqslant b \oplus c$.

(v) $a \perp b$ and $a \oplus b \leqslant c \Longleftrightarrow b \leqslant c$ and $a \leqslant c \ominus b$.

(vi) If $a \perp b$, then $a \oplus b=\left(b^{\prime} \ominus a\right)^{\prime}$.

Let $F=\left\{a_{i}: 1 \leqslant i \leqslant n\right\}$ be a finite subset of $L$. If $a_{1} \perp a_{2},\left(a_{1} \oplus a_{2}\right) \perp a_{3}$, $\ldots$ and $\left(a_{1} \oplus \cdots \oplus a_{n-1}\right) \perp a_{n}$, we say that $F$ is orthogonal and we define $\bigoplus F=$ $a_{1} \oplus \cdots \oplus a_{n}=\left(a_{1} \oplus \cdots \oplus a_{n-1}\right) \oplus a_{n}$ (by the commutative and associative laws, this sum does not depend of any permutation of elements). Now, if $A$ is an arbitrary subset of $L$ and $\mathcal{F}(A)$ is the family of all finite subsets of $A$, we say that $A$ is orthogonal if $F$ is orthogonal for every $F \in \mathcal{F}(A)$. If $A$ is orthogonal, we define $\oplus A=\bigvee\{\oplus F$ : $F \in \mathcal{F}(A)\}$, supposed that the supremum exists in $(L, \leqslant)$, and it is called the $\oplus$-sum of $A$. If $A$ is an orthogonal subset of $L$ and $B \subset A$, obviously $B$ is orthogonal too. If. moreover, there exist $\bigoplus A$ and $\oplus B$, then $\oplus B \leqslant \oplus A$. The following lemma contains more properties of orthogonal sets in an effect algebra. 
LEMMA 2.2 , Let $L$ be an effect algebra and let $\left(a_{i}\right)_{i \in I}$ be an orthogonal subset of $L$.

(i) If $I$ is finite and $J \subset I$, then $\left(\bigoplus_{i \in J} a_{i}\right) \perp\left(\bigoplus_{i \in I \backslash J} a_{i}\right)$ and

$$
\bigoplus_{i \in I} a_{i}=\left(\bigoplus_{i \in J} a_{i}\right) \oplus\left(\bigoplus_{i \in I \backslash J} a_{i}\right)
$$

(ii) If $J \subset I$ and there exist $a=\bigoplus_{i \in I} a_{i}, b=\bigoplus_{i \in J} a_{i}$ and $c=\bigoplus_{i \in I \backslash J} a_{i}$, then $b \perp c$ and $a=b \oplus c$.

(iii) If there exists $\bigoplus_{i \in M} a_{i}$ for all $M \subset I$ and $\left\{H_{j}: j \in J\right\}$ is a partition of $I$, then $A=\left\{\bigoplus_{i \in H_{j}} a_{i}: j \in J\right\}$ is an orthogonal subset of $L$, there exists $\bigoplus A$ and $\bigoplus A=\bigoplus_{i \in I} a_{i}$.

(iv) If $\left(F_{j}\right)_{j \in J}$ is a family of finite and pairwise disjoint subsets of $I$, then the set $\left\{\bigoplus_{i \in F_{j}} a_{i}: j \in J\right\}$ is orthogonal in $L$.

(v) If $b_{i} \in L$ and $b_{i} \leqslant a_{i}$ for all $i \in I$, then $\left(b_{i}\right)_{i \in I}$ is an orthogonal subset of $L$.

Proof: (i) See [16, Theorem 4.2].

(ii) Let $F_{1} \in \mathcal{F}(J)$ and $F_{2} \in \mathcal{F}(I \backslash J)$. Take $F=F_{1} \cup F_{2}$. By (i), $\left(\bigoplus_{i \in F_{1}} a_{i}\right) \perp$ $\left(\bigoplus_{i \in F_{2}} a_{i}\right)$, and $\left(\bigoplus_{i \in F_{1}} a_{i}\right) \oplus\left(\bigoplus_{i \in F_{2}} a_{i}\right)=\left(\bigoplus_{i \in F} a_{i}\right) \leqslant a$. Lemma 2.1 (v) implies that $\left(\bigoplus_{i \in F_{1}} a_{i}\right) \leqslant a \ominus\left(\bigoplus_{i \in F_{2}} a_{i}\right)$ for all $F_{1} \in \mathcal{F}(J)$, so that $b \leqslant a \ominus\left(\bigoplus_{i \in F_{2}} a_{i}\right)$. By Lemma 2.1, $\bigoplus_{i \in F_{2}} a_{i} \leqslant a \ominus b$ for all $F_{2} \in \mathcal{F}(I \backslash J)$. Hence $c \leqslant a \ominus b$, so $b \perp c$ and $b \oplus c \leqslant a$.

Conversely, if $F \in \mathcal{F}(I)$, take $F_{1}=F \cap J$ and $F_{2}=F \backslash J$. By (i) and Lemma 2.1 (iv) we have

$$
\bigoplus_{i \in F} a_{i}=\left(\bigoplus_{i \in F_{1}} a_{i}\right) \oplus\left(\bigoplus_{i \in F_{2}} a_{i}\right) \leqslant b \oplus c
$$

for all $F \in \mathcal{F}(I)$. Then $a \leqslant b \oplus c$.

(iii) Let $b_{j}=\bigoplus_{i \in H_{j}} a_{i}$ for all $j \in J$, and let $F \in \mathcal{F}(J)$. A slight refinement of the argument used in the proof of statement (ii) shows that $\left\{b_{j}: j \in F\right\}$ is an orthogonal subset of $L$ and $\bigoplus_{j \in F} b_{j} \leqslant a=\bigoplus_{i \in I} a_{i}$ for all $F \in \mathcal{F}(J)$.

Now, assume that $\bigoplus_{j \in F} b_{j} \leqslant c$ for all $F \in \mathcal{F}(J)$. Take $F_{1} \in \mathcal{F}(I)$. Then there exists $F \in \mathcal{F}(J)$ such that $F_{1} \subset \bigcup_{j \in F} H_{j}$. So $\bigoplus_{i \in F_{1}} a_{i} \leqslant \bigoplus\left\{a_{i}: i \in \bigcup_{j \in F} H_{j}\right\}$. But, 
by (ii), $\bigoplus\left\{a_{i}: i \in \bigcup_{j \in F} H_{j}\right\}=\bigoplus_{j \in F} b_{j}$. Hence $\bigoplus_{i \in F_{1}} a_{i} \leqslant c$ for every $F_{1} \in \mathcal{F}(I)$. Thus $a=\bigoplus_{i \in I} a_{i} \leqslant c$. It follows that $a=\bigvee\left\{\bigoplus_{j \in F} b_{j}: F \in \mathcal{F}(J)\right\}$ and therefore $a=\bigoplus_{j \in J} b_{j}$.

(iv) is an immediate consequence of first statement of (iii).

The proof of statement ( $v$ ) is straightforward.

A complete effect algebra is an effect algebra such that there exists $\bigoplus A$ for all orthogonal subsets $A$ of $L$. If $\bigoplus A$ exists for all countable orthogonal subsets $A$ of $L$, we say that the effect algebra $L$ is $\sigma$-complete. We say that an effect algebra $L$ is quasi- $\sigma$-complete if for every infinite countable orthogonal subset $A$ of $L$ there exists an infinite subset $M$ of $A$ such that there exists $\bigoplus N$ for all subsets $N$ of $M$. Obviously every complete effect algebra is $\sigma$-complete, and every $\sigma$-complete effect algebra is quasi- $\sigma$-complete.

It is not difficult to prove that an effect algebra $L$ is $\sigma$-complete if and only if there exists the supremum $\bigvee_{i=1}^{\infty} a_{i}$ in $(L, \leqslant)$ for all sequence $\left(a_{i}\right)_{i \in \mathbb{N}}$ in $L$ such that $a_{i} \leqslant a_{i+1}$, $i \in \mathbb{N}$.

EXAMPLE 2.3. A difference poset (or D-poset) (see [30]) is a partially ordered set $(L, \leqslant)$ with a greatest element 1 and a partial binary operation $\theta$, called a difference, such that $b \ominus a$ is defined if and only if $a \leqslant b(a, b \in L)$, and satisfying the properties (i) and (ii) of Lemma 2.1. Hence, if $(L, \perp, \oplus, 0,1)$ is an effect algebra, then $(L, \leqslant, \ominus, 1)$ is a D-poset. Conversely, if $(L, \leqslant, \ominus, 1)$ is a D-poset and we define $0=1 \ominus 1, a^{\prime}=1 \ominus a$, $a \perp b \Longleftrightarrow a \leqslant b^{\prime}$ and $a \oplus b=\left(b^{\prime} \ominus a\right)^{\prime}$ for $a \perp b$, it can be proved (see [16]) that $(L, \perp, \oplus, 0,1)$ is an effect algebra. Therefore, D-posets and effect algebras are the same thing.

EXAMPLE 2.4. Orthoalgebras are important examples of effect algebras. The definition and basic properties of orthoalgebras can be found in [17] or [39]. An effect algebra $L$ is an orthoalgebra if and only if $a \in L$ and $a \leqslant 1 \ominus a$ imply $a=0$ (see [13] or [16]). Clearly, each complete orthoalgebra (see [39]) (that is, orthosummable orthoalgebra by Habil [23]) is a complete effect algebra, and all $\sigma$-orthoalgebra (see Feldman and Wilce [15] or Habil [23]) is a $\sigma$-complete effect algebra.

EXAMPLE 2.5. Orthomodular posets are also special examples of effect algebras. In particular orthomodular lattices (and, more particularly, Boolean algebras) can be considered as effect algebras. If $(L, \perp, \oplus, 0,1)$ is an effect algebra and ' is the orthocomplementation on $L$, then $\left(L, \perp,{ }^{\prime}, 0,1\right)$ is an orthomodular poset if and only if $\{a, b, c\}$ is an orthogonal subset of $L$ whenever $a, b, c \in L$ are such that $a \perp b, b \perp c$ and $a \perp c$ (see [16]). The ( $\sigma$-)orthocomplete orthomodular posets (in particular the ( $\sigma$-)complete orthomodular lattices, and so the $(\sigma-)$ complete Boolean algebras) are relevant examples 
of $(\sigma-)$ complete effect algebras too. For more information about orthomodular posets, orthomodular lattices and Boolean algebras we refer to [29] or [41].

EXAMPLE 2.6. Let $H=(H,\langle\cdot, \cdot\rangle)$ be a Hilbert space. An operator (that is, continuous linear operator) $x$ on $H$ is said to be an effect if it is self-adjoint (that is, $x^{*}=x$, where $x^{*}$ is the adjoint operator of $x$, given by the formula $\left\langle x^{*} \varphi, \psi\right\rangle=\langle\varphi, x \psi\rangle$ for all $\varphi, \psi \in H$ ) and $0 \leqslant x \leqslant 1$, where 1 is the identity operator on $H$. If $L=\mathcal{E}(H)$ is the set of all effects on $H$, and we define, for $x, y \in L, x \perp y \Longleftrightarrow x+y \leqslant 1$, and $x \oplus y=x+y$ if $x \perp y$, then $(L, \perp, \oplus, 0,1)$ is an effect algebra which is not an orthoalgebra (see [13] or [20]). $\mathcal{E}(H)$ can be considered as the standard effect algebra. It plays an important role for unsharp measurements of quantum mechanics.

EXAMPLE 2.7. Let $H=(H,\langle\cdot, \cdot\rangle)$ be a Hilbert space over the field $\mathbb{R}$ or $\mathbb{C}$ and let $B(H)$ be the set of all bounded (that is, continuous) linear operators from $H$. An element $x \in B(H)$ is called a projection if $x$ is self-adjoint and idempotent. If $P(B(H))$ is the set of all projections of $B(H)$ and we define $x_{1} \perp x_{2} \Longleftrightarrow x_{1} x_{2}=x_{2} x_{1}=0$ and $x_{1} \oplus x_{2}=x_{1}+x_{2}$ for $x_{1} \perp x_{2}$, then $(L, \perp, \oplus, 0,1)$ is a complete effect algebra, which is also a complete orthomodular lattice, but not a Boolean algebra if dimension of $H$ is bigger or equal than 2 .

Let $L(H)$ be the set of all closed subspaces of $H$. Define $M_{1} \perp M_{2}$ if $M_{1} \cap M_{2}=$ $\{0\}$ and $M_{1} \oplus M_{2}=\bigcap\left\{M \in L(H): M \supset M_{1} \cup M_{2}\right\}$. Then $(L(H), \perp, \oplus,\{0\}, H)$ is a complete effect algebra, which is also a complete orthomodular lattice, and the map $x \rightarrow x(H)$ from $P(B(H))$ to $L(H)$ is a bijection that preserves order, orthogonality and $\oplus$-sum (see [7]). So $P(B(H))$ and $L(H)$ are isomorphic quantum logics.

This example consists of the quantum logic par excellence, and it can be considered a cornerstone in the mathematical foundations of quantum mechanics (see [35]).

EXAMPLE 2.8. Let $H=(H,\langle\cdot, \cdot\rangle)$ be a Hilbert space over $\mathbb{C}$. A von Neumann algebra on $H$ is a self-adjoint subalgebra $\mathcal{A}$ of $B(H)$ such that $\mathcal{A}=\left(\mathcal{A}^{\prime}\right)^{\prime}$, where $M^{\prime}=\{x \in$ $B(H): a x=x a$ for all $a \in M\}$ for a subset $M$ of $B(H)$. If $\mathcal{A}$ is a von Neumann algebra on $H$ and $P(\mathcal{A})=\mathcal{A} \cap P(B(H))$, then $P(\mathcal{A})$ with the partial relation $\perp$ and the binary operation $\oplus$ defined in above example is a complete effect algebra, which is also a complete orthomodular lattice. See [28] or [44] for more details concerning von Neumann algebras.

EXAMPLE 2.9. Let $(G,+, 0, \leqslant)$ be a partially ordered commutative group, and let $u \in G$ be such that $0 \leqslant u$ and $0 \neq u$. Take $L=\{x \in G: 0 \leqslant x \leqslant u\}$. For $x, y \in L$, let us define $x \perp y \Longleftrightarrow x+y \in L$, and $x \oplus y=x+y$ if $x \perp y$. Then $(L, \perp, \oplus, 0, u)$ is an effect algebra and it is called an interval effect algebra (see [16]) which it is not, in general, an orthoalgebra. For instance, if $G=\mathbb{R}$ with the usual ordering and $u=1$, then $([0,1], \perp, \oplus, 0,1)$ is an effect algebra but not an orthoalgebra 
because $1 / 2 \leqslant 1 \ominus 1 / 2$ and $1 / 2 \neq 0$ (see Example 2.4).

For more details concerning effect algebras we refer to [16]. Other interesting examples on effect algebras, as well as quantum logics in general, can be found in [14, $20,22,25,30]$.

\section{Group-Valued measures on efFect algebras}

For the remainder of this paper, $L=(L, \perp, \oplus, 0,1)$ will be an effect algebra and $G=(G,+, 0, \tau)$ will be a Hausdorff topological commutative group (see Higgins [27]). Let $\mathcal{N}(G)$ denote the set of all neighbourhoods of 0 in $G$. It is well known that $(G, \tau)$ is a regular topological space and so the closed neighbourhoods of 0 form a base of $\mathcal{N}(G)$ (see [27]). The definitions of convergence and the Cauchy condition of a sequence or a net in a topological group are standard. By convergence of a series in a topological group we refer, as usual, to convergence of the sequence of partial sums.

A $G$-valued measure on $L$ is an element $\mu \in G^{L}$ such that $\mu(a \oplus b)=\mu(a)+\mu(b)$ for all pairs of elements $a, b \in L$ with $a \perp b$. Every $G$-valued measure on $L$ is finitely additive, that is, $\mu\left(a_{1} \oplus \cdots \oplus a_{n}\right)=\mu\left(a_{1}\right)+\cdots+\mu\left(a_{n}\right)$ whenever $\left\{a_{1}, \ldots, a_{n}\right\}$ is an orthogonal finite subset of $L$. The set of all $G$-valued measures on $L$ will be denoted by $\mathrm{a}(L, G)$. If $\mu \in \mathrm{a}(L, G)$, then $\mu(0)=0$ and $\mu\left(a^{\prime}\right)=\mu(1)-\mu(a)$ for all $a \in L$. Moreover if $\mu \in G^{L}$, then $\mu \in \mathrm{a}(L, G)$ if and only if $\mu(b \ominus a)=\mu(b)-\mu(a)$ for all $a, b \in L$ with $a \leqslant b$.

Let $\mu \in \mathrm{a}(L, G)$. We say that $\mu$ is $s$-bounded if for every orthogonal sequence $\left(a_{n}\right)_{n \in \mathbb{N}}$ in $L,\left(\mu\left(a_{n}\right)\right)_{n \in \mathbb{N}}$ converges to 0 in the topology of $G$. We say that $\mu$ is $\sigma$-additive if for every orthogonal sequence $\left(a_{n}\right)_{n \in \mathbb{N}}$ in $L$ such that $a=\bigoplus_{n \in \mathbb{N}} a_{n}$ exists in $L$, the series $\sum_{n=1}^{\infty} \mu\left(a_{n}\right)$ converges to $\mu(a)$. The subsets of $\mathrm{a}(L, G)$ containing all $G$ valued $\sigma$-additive or $s$-bounded measures on $L$ will be denoted by $\operatorname{ca}(L, G)$ or $\operatorname{sa}(L, G)$ respectively.

Lemma 3.1. If $L$ is quasi $\sigma$-complete and $\mu \in \operatorname{ca}(L, G)$, then $\mu \in \operatorname{sa}(L, G)$.

Proof: Suppose the contrary. Then there exist an orthogonal sequence $\left(a_{n}\right)_{n \in \mathbb{N}}$ in $L$, a neighbourhood $U \in \mathcal{N}(G)$ and an infinite subset $M$ of $\mathbb{N}$ such that $\mu\left(a_{n}\right) \notin U$ for all $n \in M$. By the quasi $\sigma$-completeness of $L$, there exists an infinite subset $N$ of $M$ for which there exists $a=\bigoplus_{n \in N} a_{n}$. Since $\mu$ is countably additive, the series $\sum_{n \in N} \mu\left(a_{n}\right)$ converges to $\mu(a)$. Then the sequence $\left(\mu\left(a_{n}\right)\right)_{n \in N}$ converges to 0 , a contradiction.

Let us note that the inclusion $\operatorname{ca}(L, G) \subset \operatorname{sa}(L, G)$ is not true if $L$ is an arbitrary effect algebra (see [10] for a counterexample). The converse inclusion is also false, even if $L$ is a complete effect algebra, as we can see from the following example.

EXAmPLE 3.2. Let $H$ be an infinite-dimensional separable Hilbert space and let $\left\{\varphi_{n}\right.$ : 
$n \in \mathbb{N}\}$ be an orthonormal basis of $H$. Let LIM be a Banach limit on $l^{\infty}$ (see [43]) and let $\mu: P(B(H)) \rightarrow \mathbb{R}$ defined by the formula: $\mu(e)=\operatorname{LIM}\left\langle e \varphi_{n}, \varphi_{n}\right\rangle$. Clearly, $\mu$ is an $\mathbb{R}$-valued measure on the complete effect algebra $P(B(H))$. Let $p_{n}$ be the projection onto the subspace spanned by $\varphi_{n}$. Then $\left(p_{n}\right)_{n \in \mathbb{N}}$ is an orthogonal sequence in $P(B(H))$ such that $\mu\left(p_{n}\right)=0$ for all $n \in \mathbb{N}$. Moreover, $1=\oplus p_{n}$. But $\mu(1)=1 \neq 0$. Therefore, $\mu$ is not $\sigma$-additive. On the other hand, if $\left(e_{n}\right)_{n \in \mathbb{N}}$ is an orthogonal sequence in $L$ and $e=\bigoplus_{n \in \mathbb{N}} e_{n}$, then $\sum_{i=1}^{n} \mu\left(e_{i}\right) \leqslant \mu(e)$ for all $n$. Hence the series of non-negative real numbers $\sum_{n=1}^{\infty} \mu\left(e_{n}\right)$ is convergent, and so the sequence $\left(\mu\left(e_{n}\right)\right)_{n \in \mathbb{N}}$ converges to 0 . Therefore, $\mu$ is $s$-bounded.

It is possible to give different definitions of $s$-bounded measures. Thanks to the following lemma, several of these definitions are equivalent:

Lemma 3.3. A measure $\mu \in \mathrm{a}(L, G)$ is $s$-bounded if and only if for every orthogonal family $\left(a_{i}\right)_{i \in I}$ and every $V \in \mathcal{N}(G)$ there exists a finite subset $F_{0} \subset I$ such that

$$
\left\{\sum_{i \in F} \mu\left(e_{i}\right): F \in \mathcal{F}\left(I \backslash F_{0}\right), e_{i} \leqslant a_{i} \forall i \in F\right\} \subset V .
$$

Proof: Obviously, the condition implies the $s$-boundedness of $\mu$. Suppose that $\mu$ is $s$-bounded and the condition is false. Then we can find an orthogonal family $\left(a_{i}\right)_{i \in I}$ in $L$ (not finite) and $V \in \mathcal{N}(G)$ such that for all $F_{0} \in \mathcal{F}(I)$ there exist $F \in \mathcal{F}\left(I \backslash F_{0}\right)$ and $\left\{e_{i}: i \in F\right\}$, with $e_{i} \leqslant a_{i}$ for $i \in F$ and $\sum_{i \in F} \mu\left(e_{i}\right) \notin V$.

Starting with $F_{0}=\emptyset$, we take a sequence of disjoint sets $F_{1}, F_{2}, \ldots$, and elements $e_{i}$ with $e_{i} \leqslant a_{i}$ for $i \in F_{k}$ and $k \in \mathbb{N}$, such that $\sum_{i \in F_{k}} \mu\left(e_{i}\right) \notin V$ for all $k \in \mathbb{N}$. Since $\left(a_{i}\right)_{i \in I}$ is orthogonal and $e_{i} \leqslant a_{i}$, by Lemma $2.1(\mathrm{v})$, the set $\left\{e_{i}: i \in \cup\left\{F_{k}: k \in \mathbb{N}\right\}\right\}$ is orthogonal in $L$. Let $b_{k}=\bigoplus\left\{e_{i}: i \in F_{k}\right\}$ for $k \in \mathbb{N}$. Then, by Lemma 2.1 (iv), $\left(b_{k}\right)_{k \in \mathbb{N}}$ is an orthogonal sequence in $L$ such that

$$
\mu\left(b_{k}\right)=\mu\left(\bigoplus_{i \in F_{k}} e_{i}\right)=\sum_{i \in F_{k}} \mu\left(e_{i}\right) \notin V
$$

for any $k \in \mathbb{N}$. This contradicts the $s$-boundedness of $\mu$.

Corollary 3.4. For $\mu \in \mathrm{a}(L, G)$ the following conditions are equivalent:

(i) $\mu$ is $s$-bounded.

(ii) $\mu$ is strongly additive, that is, for every orthogonal sequence $\left(a_{n}\right)_{n \in \mathbb{N}}$ in $L$, the series $\sum_{n=1}^{\infty} \mu\left(a_{n}\right)$ satisfies the Cauchy condition. 
(iii) For every orthogonal sequence $\left(a_{n}\right)_{n \in \mathbb{N}}$ in $L$ and every $V \in \mathcal{N}(G)$ there exists $n_{0} \in \mathbb{N}$ such that $\left\{\mu(e): e \leqslant a_{i}, i \geqslant n_{0}\right\} \subset V$.

(iv) For every orthogonal family $\left(a_{i}\right)_{i \in I}$ in $L$ and every $V \in \mathcal{N}(G)$ there exists $F_{0} \in \mathcal{F}(I)$ such that $\mu\left(a_{i}\right) \in V$ for all $i \in I \backslash F_{0}$.

(v) For every orthogonal family $\left(a_{i}\right)_{i \in I}$ in $L$ and every $V \in \mathcal{N}(G)$ there exists $F_{0} \in \mathcal{F}(I)$ such that $\sum_{i \in F} \mu\left(a_{i}\right) \in V$ for all $F \in \mathcal{F}\left(I \backslash F_{0}\right)$.

(vi) For every orthogonal family $\left(a_{i}\right)_{i \in I}$ in $L$ and every $V \in \mathcal{N}(G)$ there exists $F_{0} \in \mathcal{F}(I)$ such that $\left\{\mu(e): e \leqslant a_{i}, i \in I \backslash F_{0}\right\} \subset V$.

PROOF: It is immediate to prove that each of the conditions (ii) to (vi) implies the $s$-boundedness of $\mu$, and, conversely, they are implied by the condition of the previous lemma.

The following lemma will be used in the proof of the Brooks-Jewett theorem and other main results included in the next section:

LEMma 3.5. Let $L$ be a quasi- $\sigma$-complete effect algebra and let $\left\{\mu_{n}: n \in \mathbb{N}\right\} \subset$ $\mathrm{sa}(L, G)$. If $\left(\mu_{n}(a)\right)_{n \in \mathbb{N}}$ is a Cauchy sequence for all $a \in L$ and $\left(a_{i}\right)_{i \in I}$ is an orthogonal family in $L$, then the net $\left(\sum_{i \in F} \mu_{n}\left(a_{i}\right)\right)_{F \in \mathcal{F}(I)}$ is Cauchy uniformly in $n \in \mathbb{N}$.

Proof: Suppose the contrary. Then we can take an orthogonal family $\left(a_{i}\right)_{i \in I}$ in $L$ and $U \in \mathcal{N}(G)$ such that for all $F_{0} \in \mathcal{F}(I)$ the set

$$
\left\{\sum_{i \in F} \mu_{n}\left(a_{i}\right): F \in \mathcal{F}\left(I \backslash F_{0}\right)\right\} \not \subset U
$$

for some $n \in \mathbb{N}$.

First, we can see that (3.1) will be valid for an infinite number of $n \in \mathbb{N}$. In fact, if $\left\{\sum_{i \in F} \mu_{n}\left(a_{i}\right): F \in \mathcal{F}\left(I \backslash F_{0}\right)\right\} \subset U$ for $n \in \mathbb{N} \backslash\left\{n_{1}, \ldots, n_{k}\right\}$, since $\mu_{n_{1}}, \ldots, \mu_{n_{k}} \in$ $\operatorname{sa}(L, G)$, there exist sets $F_{1}, \ldots, F_{k} \in \mathcal{F}(I)$ such that $\left\{\sum_{i \in F} \mu_{n_{j}}\left(a_{i}\right): F \in \mathcal{F}\left(I \backslash F_{j}\right)\right\} \subset$ $U$ for $j=1 \ldots k$. Putting $H=F_{0} \cup F_{1} \cup \cdots \cup F_{k}$ it follows that $\left\{\sum_{i \in F} \mu_{n}\left(a_{i}\right): F \in\right.$ $\mathcal{F}(I \backslash H)\} \subset U$ for all $n \in \mathbb{N}$, which contradicts (3.1).

So, we have proved that for every $F_{0} \in \mathcal{F}(I)$ and every $n_{0} \in \mathbb{N}$ there exist $F \in$ $\mathcal{F}\left(I \backslash F_{0}\right)$ and $n>n_{0}$ such that $\sum_{i \in F} \mu_{n}\left(a_{i}\right) \notin U$. Let us fix a symmetric neighbourhood $V \in \mathcal{N}(G)$ such that $U \supset V+V+V+V(=4 V)$.

Take $F_{0}=F_{0}^{\prime}=\emptyset$ and $n_{0}=0$. There exist $n_{1}>0$ and $F_{1} \in \mathcal{F}(I)$ satisfying that $\sum_{i \in F_{1}} \mu_{n_{1}}\left(a_{i}\right) \notin U$. Since $\mu_{n_{1}}$ is $s$-bounded, by Corollary 3.4, there exists $F_{1}^{\prime} \in \mathcal{F}(I)$, which we can consider $F_{1}^{\prime} \supset F_{1}\left(=F_{1} \cup F_{0}^{\prime}\right)$, such that $\sum_{i \in F} \mu_{n_{1}}\left(a_{i}\right) \in V$ for each $F \in$ 
$\mathcal{F}\left(I \backslash F_{1}^{\prime}\right)$. But $\left(\mu_{n}\left(\bigoplus_{i \in F_{1}} a_{i}\right)\right)_{n \in \mathbb{N}}$ is a Cauchy sequence. Then there exists $n_{1}^{\prime}>n_{1}$ such that $\sum_{i \in F}\left(\mu_{n}\left(a_{i}\right)-\mu_{m}\left(a_{i}\right)\right) \in V$ for every $n, m \geqslant n_{1}^{\prime}$ and every $F \subset F_{1}$.

Now, from $n_{1}^{\prime}$ and $F_{1}^{\prime}$, it is possible to find $F_{2} \in \mathcal{F}\left(I \backslash F_{1}^{\prime}\right)$ and $n_{2}>n_{1}^{\prime}$ such that $\sum_{i \in F_{2}} \mu_{n_{2}}\left(a_{i}\right) \notin U$. Following the construction, it is easy to prove, by induction, the existence of a sequence $\left(F_{k}\right)_{k \in \mathbb{N}}$ of pairwise disjoint finite subsets of $I$ and an increasing sequence $\left(n_{k}\right)_{k \in \mathbb{N}}$ satisfying the properties:

$$
\sum_{i \in F_{k}} \mu_{n_{k}}\left(a_{i}\right) \notin U, \quad \sum_{i \in F_{k+1}} \mu_{n_{k}}\left(a_{i}\right) \in V
$$

and

$$
\sum_{i \in F}\left(\mu_{n}\left(a_{i}\right)-\mu_{m}\left(a_{i}\right)\right) \in V \quad \text { for all } n, m \geqslant n_{k} \text { and } F \subset \bigcup_{j<k} F_{j}
$$

Put $\eta_{k}=\mu_{n_{k}}-\mu_{n_{k-1}}$ for $k \geqslant 2$. Then $\eta_{k} \in \mathrm{sa}(L, G)$ for all $k$ and the sequence $\left(\eta_{k}(a)\right)_{k \in \mathbb{N}}$ converges to 0 for each $a \in L$. Moreover, by (3.2),

$$
\sum_{i \in F_{k}} \eta_{k}\left(a_{i}\right)=\sum_{i \in F_{k}}\left(\mu_{n_{k}}\left(a_{i}\right)-\mu_{n_{k-1}}\left(a_{i}\right)\right)=\sum_{i \in F_{k}} \mu_{n_{k}}\left(a_{i}\right)-\sum_{i \in F_{k}} \mu_{n_{k-1}}\left(a_{i}\right) \notin 3 V .
$$

Let $b_{k}=\bigoplus_{i \in F_{k}} a_{i}$ for all $k \in \mathbb{N}$. By Lemma 2.2 (iv), $\left(b_{k}\right)_{k \in \mathbb{N}}$ is an orthogonal sequence in $L$. By the quasi- $\sigma$-completeness of $L$, we can take a subsequence $\left(b_{k_{n}}\right)_{n \in \mathbb{N}}$ such that there exists $\bigoplus_{n \in M} b_{k_{n}}$ for every subset $M$ of $\mathbb{N}$. Let us place the sequence $\left(b_{k_{n}}\right)_{n \in \mathbb{N}}$ as a double sequence, namely $\left(b_{j l}\right)_{j, l \in \mathbb{N}}$. Then for every $l \in \mathbb{N}$ there exists $d_{l}=\bigoplus_{j \in \mathbb{N}} b_{j l}$ in $L$ and, by Lemma 2.2 (iii), $\left(d_{l}\right)_{l \in \mathbb{N}}$ is an orthogonal sequence in $L$. Since $\eta_{k_{1}}$ is $s$-bounded, using Corollary 3.4 , it is possible to find $l_{1} \in \mathbb{N}$ such that $\eta_{k_{1}}(e) \in V$ whenever $e \leqslant d_{l}$ and $l \geqslant l_{1}$. Reordering, if necessary, we can consider the sequence $\left(b_{j l_{1}}\right)_{j \in \mathbb{N}}$ as a subsequence $\left(b_{j}^{1}\right)_{j \in \mathbb{N}}$ of $\left(b_{k_{n}}\right)_{n \in \mathbb{N}}$. Let $r_{1}$ be the least $k_{n} \in \mathbb{N}$ such that $b_{k_{n}} \in\left\{b_{j}^{1}: j \in \mathbb{N}\right\}$, that is, $b_{r_{1}}=b_{1}^{1}$. If we consider now $\left(b_{j}^{1}\right)_{j \geqslant 2}$ as a double sequence, with a reasoning as above, we can find a subsequence $\left(b_{j}^{2}\right)_{j \in \mathbb{N}}$ of $\left(b_{j}^{1}\right)_{j \geqslant 2}$ and $r_{2}>r_{1}$ such that $\eta_{r_{1}}(e) \in V$ whenever $e \leqslant \bigoplus_{j \in \mathbb{N}} b_{j}^{2}$. Now put $b_{r_{2}}=b_{1}^{2}$. In this way we get, for every $k \in \mathbb{N}$, an orthogonal sequence $\left(b_{j}^{k}\right)_{j \in \mathbb{N}}$ in $L$ and $r_{k} \in \mathbb{N}$ such that $b_{r_{k}}=b_{1}^{k},\left(b_{j}^{k}\right)_{j \in \mathbb{N}}$ is a subsequence of $\left(b_{j}^{k-1}\right)_{j \geqslant 2}$ and $\eta_{r_{k}}(e) \in V$ whenever $e \leqslant \bigoplus_{j \in \mathbb{N}} b_{j}^{k+1}$. But $\bigoplus_{j>k} b_{r_{j}} \leqslant \bigoplus_{j \in \mathbb{N}} b_{j}^{k+1}$ for all $k \in \mathbb{N}$. Then $\eta_{r_{k}}\left(\bigoplus_{j>k} b_{r_{j}}\right) \in V$. We have 
also that $\eta_{r_{k}}\left(b_{r_{k}}\right) \notin 3 V$ by (3.4) and $\eta_{r_{k}}\left(\bigoplus_{j<k} b_{r_{j}}\right) \in V$ by (3.3). On the other hand, from Lemma 2.2 , it follows that

$$
\bigoplus_{k \in \mathbb{N}} b_{r_{k}}=b_{r_{k}} \oplus\left(\bigoplus_{j>k} b_{r_{j}}\right) \oplus\left(\bigoplus_{j<k} b_{r_{j}}\right) .
$$

Then $\eta_{r_{k}}\left(\bigoplus_{k \in \mathbb{N}} b_{r_{k}}\right) \notin V$ for every $k \in \mathbb{N}$. A contradiction, because $\left(\eta_{r_{k}}(a)\right)_{k \in \mathbb{N}}$ converges to 0 for every $a \in L$.

\section{Main Results}

We are in position to obtain the main results of this paper. Let us start with a non-commutative version of the Brooks-Jewett theorem.

Throughout this section we assume that $L$ is a quasi- $\sigma$-complete effect algebra.

Let $M$ be a subset of $\operatorname{sa}(L, G)$. We say that $M$ is uniformly $s$-bounded if for every orthogonal sequence $\left(a_{n}\right)_{n \in \mathbb{N}}$ in $L$ and every $V \in \mathcal{N}(G)$ there exists $n_{0} \in \mathbb{N}$ such that $\left\{\mu\left(a_{n}\right): n \geqslant n_{0}, \mu \in M\right\} \subset V$. In the same natural way may be defined the uniform countable additivity.

The following theorem generalises the original result of Brooks and Jewett [5] as well as [46, Theorem 4.1] and, essentially, a theorem of [38].

Theorem 4.1. (Brooks-Jewett Theorem.) Let $\left\{\mu_{n}: n \in \mathbb{N}\right\} \subset \operatorname{sa}(L, G)$. If, for every $a \in L,\left(\mu_{n}(a)\right)_{n \in \mathbb{N}}$ is a Cauchy sequence in $G$, then $\left\{\mu_{n}: n \in \mathbb{N}\right\}$ is uniformly $s$-bounded. Moreover, if $\left(\mu_{n}(a)\right)_{n \in \mathbb{N}}$ converges to $\mu(a)$ for all $a \in L$, then $\mu \in \mathrm{sa}(L, G)$.

Proof: Let $\left(a_{i}\right)_{i \in \mathbb{N}}$ be an orthogonal sequence in $L$. Putting $I=\mathbb{N}$ in Lemma 3.5 it follows that the series $\sum_{i=1}^{\infty} \mu_{n}\left(a_{i}\right)$ is Cauchy uniformly in $n \in \mathbb{N}$. So $\lim _{i \rightarrow \infty} \mu_{n}\left(a_{i}\right)=0$ uniformly in $n \in \mathbb{N}$ and $\left\{\mu_{n}: n \in \mathbb{N}\right\}$ is uniformly $s$-bounded. Now, suppose that $\lim _{n \rightarrow \infty} \mu_{n}(a)=\mu(a)$ for every $a \in L$, and let $V$ be a closed neighbourhood of $\mathcal{N}(G)$. Then there exists $i_{0} \in \mathbb{N}$ such that $\left\{\mu_{n}\left(a_{i}\right): i \geqslant i_{0}, n \in \mathbb{N}\right\} \subset V$. Then $\left\{\mu\left(a_{i}\right): i \geqslant\right.$ $\left.i_{0}\right\} \subset V$ and $\mu$ is $s$-bounded.

In the setting of non-commutative measures the study of completely additive measures is very important.

If $M \subset \mathrm{a}(L, G)$, we say that $M$ is uniformly completely additive if for every orthogonal family $\left(a_{i}\right)_{i \in I}$ in $L$ such that $a=\bigoplus_{i \in I} a_{i}$ exists in $L$ and every $V \in \mathcal{N}(G)$, there exists $F_{0} \in \mathcal{F}(I)$ such that $\mu(a)-\sum_{i \in F} \mu\left(a_{i}\right) \in V$ for every $F \in \mathcal{F}(I)$ with 
$F \supset F_{0}$ and every $\mu \in M$. If $\mu \in \mathrm{a}(L, G)$ and $\{\mu\}$ is uniformly completely additive, we say that $\mu$ is completely additive and we write $\mu \in \operatorname{cca}(L, G)$. It is clear that $\operatorname{cca}(L, G) \subset \mathrm{ca}(L, G)$, and, in general, the inclusion is strict.

For completely additive measures we can give the following result:

ThEOREM 4.2. Let $\left\{\mu_{n}: n \in \mathbb{N}\right\} \subset \operatorname{cca}(L, G)$. If $\left(\mu_{n}(a)\right)_{n \in \mathbb{N}}$ is a Cauchy sequence in $G$ for all $a \in L$, then $\left\{\mu_{n}: n \in \mathbb{N}\right\}$ is uniformly completely additive. Moreover, if $\left(\mu_{n}(a)\right)_{n \in \mathbb{N}}$ converges to $\mu(a)$ for all $a \in L$, then $\mu \in \operatorname{cca}(L, G)$.

ProOF: Let $\left(a_{i}\right)_{i \in I}$ be an orthogonal family such that there exists $a=\bigoplus_{i \in I} a_{i}$ in $L$, and let $U \in \mathcal{N}(G)$. Take $V \in \mathcal{N}(G)$ such that $V+V \subset U$. By Lemma 3.5, there exists $F_{0} \in \mathcal{F}(I)$ such that $\sum_{i \in F} \mu_{n}\left(a_{i}\right) \in V$ for every $F \in \mathcal{F}\left(I \backslash F_{0}\right)$ and every $n \in \mathbb{N}$. Let us fix $J \in \mathcal{F}(I)$ such that $J \supset F_{0}$ and $n \in \mathbb{N}$. Since $\mu_{n}$ is completely additive, there is $F_{n} \in \mathcal{F}(I)$ such that $\mu_{n}(a)-\sum_{i \in F} \mu_{n}\left(a_{i}\right) \in V$ for all $F \supset F_{n}$. Then

$$
\mu_{n}(a)-\sum_{i \in J} \mu_{n}\left(a_{i}\right)=\mu_{n}(a)-\sum_{i \in J \cup F_{n}} \mu_{n}\left(a_{i}\right)+\sum_{i \in F_{n} \backslash J} \mu_{n}\left(a_{i}\right) \in V+V \subset U
$$

which shows that $\left\{\mu_{n}: n \in \mathbb{N}\right\}$ is uniformly completely additive.

The second statement of theorem can be proved as in Theorem 4.1.

The last result can be considered as a generalisation of classical Nikodým convergence theorem (called also Vitali-Hahn-Saks-Nikodým theorem). Indeed, if we take $I=\mathbb{N}$ in the proof of Theorem 4.2, we get the following corollary, which contains, among others, [8, Theorem 4], [4, Corollary 3.8], [3, (6.4)] and [24, Theorem 4.10].

Corollary 4.3. (Nikodým Convergence Theorem.) Let $\left\{\mu_{n}: n \in \mathbb{N}\right\} \subset$ $\operatorname{ca}(L, G)$. If $\left(\mu_{n}(a)\right)_{n \in \mathbb{N}}$ is a Cauchy sequence in $G$ for all $a \in L$, then $\left\{\mu_{n}: n \in \mathbb{N}\right\}$ is uniformly countably additive. Moreover, if $\left(\mu_{n}(a)\right)_{n \in \mathbb{N}}$ converges to $\mu(a)$ for all $a \in L$, then $\mu \in \operatorname{ca}(L, G)$.

As a consequence of Theorem 4.2 , it is possible to generalise the Vitali-HahnSaks theorem for von Neumann algebras due to Aarnes [1]. At the same time, in the next corollary we shall extend similar results contained in [9] and [31]. Let $\mathcal{A}$ be a von Neumann algebra and let $P(\mathcal{A})$ be the set of all projections of $\mathcal{A}$ (see Example 2.8). Let $\varphi$ be a homomorphism from $\mathcal{A}$ into $G$. We shall say that $\varphi$ is normal if $\left.\varphi\right|_{P(\mathcal{A})} \in \operatorname{cca}(P(\mathcal{A}), G)$ (see [28, Theorem 7.1.12]).

Corollary 4.4. (Nikodým Convergence Theorem.) Let $\left\{\varphi_{\mathfrak{n}}: n \in \mathbb{N}\right\}$ be a sequence of normal homomorphisms from a von Neumann algebra $\mathcal{A}$ into $G$, and assume that the sequence $\left(\varphi_{n}(p)\right)_{n \in \mathbb{N}}$ converges to $\varphi(p)$ for every projection $p \in P(\mathcal{A})$. Then $\varphi$ is a normal homomorphism from $\mathcal{A}$ into $G$. 
It is possible to obtain a new convergence theorem for $\tau$-smooth measures. Let $M \subset \mathrm{a}(L, G)$. We say that $M$ is uniformly $\tau$-smooth if for every filtering downwards set $D \subset L$ such that $\bigwedge D=0$ and every $V \in \mathcal{N}(G)$ there exists $a_{0} \in D$ such that $\mu(a) \in V$ whenever $a \in D$ with $a \leqslant a_{0}$ and $\mu \in M$. A measure $\mu \in \mathrm{a}(L, G)$ is $\tau$-smooth if the set $\{\mu\}$ is uniformly $\tau$-smooth.

Lemma 4.5. If $\mu \in \mathrm{a}(L, G)$ is $\tau$-smooth, then $\mu$ is completely additive.

Proof: Let $\left(a_{i}\right)_{i \in I}$ be an orthogonal family in $L$ such that $a=\bigoplus_{i \in I} a_{i}$ exists in $L$ and let $V \in \mathcal{N}(G)$. For $F \in \mathcal{F}(I)$ we write $d_{F}=a \ominus\left(\bigoplus_{i \in F} a_{i}\right)$. By Lemma 2.1 (ii), the family $\left\{d_{F}: F \in \mathcal{F}(I)\right\}$ is filtering downwards. Let $b \in L$ such that $b \leqslant d_{F}$ for all $F \in \mathcal{F}(I)$. Then $b \leqslant a$ and, by Lemma 2.1, $a \ominus d_{F}=\bigoplus_{i \in F} a_{i} \leqslant a \ominus b$ for all $F \in \mathcal{F}(I)$. So $a \leqslant a \ominus b$ and $b=0$. Then we have proved that $\bigwedge\left\{d_{F}: F \in \mathcal{F}(I)\right\}=0$. Since $\mu$ is $\tau$-smooth, there exists $F_{0} \in \mathcal{F}(I)$ such that $\mu\left(d_{F}\right) \in V$ for all $F \supset F_{0}$. It follows that $\mu(a)-\sum_{i \in F} \mu\left(a_{i}\right) \in V$ for all $F \supset F_{0}$. Therefore $\mu$ is completely additive.

The following convergence theorem for $\tau$-smooth measures was unknown, even in the setting of classical measures defined on $\sigma$-complete Boolean algebras.

THEOREM 4.6. Let $\left(\mu_{n}\right)_{n \in \mathbb{N}}$ be a sequence of $\tau$-smooth measures from $L$ into $G$. If $\left(\mu_{n}(a)\right)_{n \in \mathbb{N}}$ is a Cauchy sequence in $G$ for all $a \in L$, then $\left\{\mu_{n}: n \in \mathbb{N}\right\}$ is uniformly $\tau$-smooth. Moreover, if $\left(\mu_{n}(a)\right)_{n \in \mathbb{N}}$ converges to $\mu(a)$ for all $a \in L$, then $\mu$ is $\tau$-smooth.

Proof: Suppose that $\left\{\mu_{n}: n \in \mathbb{N}\right\}$ is not uniformly $\tau$-smooth. Then there exists a filtering downwards subset $D$ of $L$ with $\wedge D=0$ and $U \in \mathcal{N}(G)$ such that for each $a \in D$ there is $b \in D, b \leqslant a$, with $\mu_{n}(b) \notin U$ for some $n \in \mathbb{N}$. It is easy to verify that the set $\left\{n \in \mathbb{N}: \mu_{n}(b) \notin U\right\}$ is not finite. Then, for every $a \in D$ and $n \in \mathbb{N}$ there are $m>n$ and $b \in D, b \leqslant a$, such that $\mu_{n}(b) \notin U$.

Let $V \in \mathcal{N}(G)$ such that $V+V \subset U$. Starting with $n_{1}=0$ and $a_{1} \in D$ arbitrary, let $a_{2} \leqslant a_{1}$ and $n_{2}>n_{1}$ such that $\mu_{n_{2}}\left(a_{2}\right) \notin U$. Since $\mu_{n_{2}}$ is $\tau$-smooth, it is possible to find $b_{2} \in D, b_{2} \leqslant a_{2}$, such that $\mu_{n_{2}}(a) \in V$ for all $a \in D$ with $a \leqslant b_{2}$. Take $n_{3}>n_{2}$ and $a_{3} \in D, a_{3} \leqslant b_{2}$, such that $\mu_{n_{3}}\left(a_{3}\right) \notin U$. Following in this way, we get a sequence $\left(a_{k}\right)_{k \in \mathbb{N}}$ of elements of $D$ and a sequence $\left(n_{k}\right)_{k \in \mathbb{N}}$ in $\mathbb{N}$ satisfying: $\mu_{n_{k}}\left(a_{k}\right) \notin U, \mu_{n_{k}}\left(a_{k+1}\right) \in V, a_{k+1} \leqslant a_{k}$ and $n_{k+1}>n_{k}$ for all $k \in \mathbb{N}$.

Let $d_{k}=a_{k} \ominus a_{k+1}$. By Lemma 2.1 (ii) and (v) it follows that $d_{1} \perp d_{2}$ and $d_{1} \oplus d_{2}=a_{1} \ominus a_{3}$. A simple reasoning by induction shows that $\left\{d_{1}, \ldots, d_{n}\right\}$ is orthogonal and $d_{1} \oplus \cdots \oplus d_{n}=a_{1} \ominus a_{n+1}$ for all $n \in \mathbb{N}$. Then $\left(d_{k}\right)_{k \in \mathbb{N}}$ is an orthogonal sequence in $L$ such that $\mu_{n_{k}}\left(d_{k}\right) \notin V$ for all $k \in \mathbb{N}$. But by Lemma 4.5 , each measure $\mu_{n}$ is completely additive and, by Lemma 3.1, s-bounded. Applying the Brooks-Jewett 
Theorem 4.1, it follows that $\left\{\mu_{n}: n \in \mathbb{N}\right\}$ is uniformly $s$-bounded. This contradicts the fact that $\mu_{n_{k}}\left(d_{k}\right) \notin V$ for all $k \in \mathbb{N}$. So, the proof of first part of theorem is complete.

The proof of second statement is standard.

REMARK 4.7. It is usual to say that $\mu \in \mathrm{a}(L, G)$ is order continuous if $\left(\mu\left(a_{n}\right)\right)_{n \in \mathbb{N}}$ converges to 0 for all decreasing sequence $\left(a_{n}\right)_{n \in \mathbb{N}}$ in $L$ such that $\wedge a_{n}=0$. With a proof similar to that of Lemma 4.5 it can be proved that $\mu$ is order continuous if and only if $\mu$ is countably additive (see [8, Proposition 2]). Therefore, the Nikodým convergence theorem (Corollary 4.3) can be also obtained as a consequence of Theorem 4.6 .

Now, we shall give a convergence theorem for regular measures. But, firstly, we need some preliminary definitions.

We say that an element $a \in L$ is principal if $p, q \in L, p \perp q$ and $p, q \leqslant a$ imply $p \oplus q \leqslant a$. We say that $a \in L$ is central if $a$ and $a^{\prime}$ are principal and for all $p \in L$ there exist $q, r \in L$ such that $q \leqslant a, r \leqslant a^{\prime}$ and $q \oplus r=p$. The set of all central elements of $L$ is called the centre of $L$, and it is denoted by $C(L)$. Greechie, Foulis and Pulmannová [21, Theorem 5.4] have shown that the centre $C(L)$ is a sub-effect algebra of $L$ and, as an effect algebra in its own right, $C(L)$ forms a Boolean algebra. Furthermore, if $a, b \in C(L)$, there exists the supremum $a \vee b$ in $C(L)$, which is also the supremum of $a$ and $b$ as calculated in $L$. As a consequence, if $a, b \in C(L)$ and $a \perp b$, then $a \vee b$ is principal. So, $a \oplus b \leqslant a \vee b$, which implies that $a \vee b=a \oplus b$.

For more information about principal and central elements in an effect algebra we refer to [21].

A paving in $L$ is a subset $K$ of $L$ such that $0 \in K$ and the supremum $a \vee b$ exists in $L$ and belongs to $K$ for all $a, b \in K$.

Let $K$ be a paving in $L$ and let $M \subset \mathrm{a}(L, G)$. We say that $M$ is uniformly $K$-regular if for every $U \in \mathcal{N}(G)$ and every $a \in L$ there exists $b \in K, b \leqslant a$, such that $\mu(c) \in U$ whenever $c \in L$ with $c \leqslant a \ominus b$ and $\mu \in M$. A measure $\mu \in \mathrm{a}(L, G)$ is $K$-regular if the set $\{\mu\}$ is uniformly $K$-regular.

THEOREM 4.8. Let $K$ be a paving in $L$ such that $K \subset C(L)$, and let $\left(\mu_{n}\right)_{n \in N}$ be a sequence of $K$-regular and $s$-bounded measures from $L$ into $G$. If $\left(\mu_{n}(a)\right)_{n \in \mathrm{N}}$ is a Cauchy sequence in $G$ for all $a \in L$, then $\left\{\mu_{n}: n \in \mathbb{N}\right\}$ is uniformly $K$-regular. Moreover, if $\left(\mu_{n}(a)\right)_{n \in \mathbb{N}}$ converges to $\mu(a)$ for all $a \in L$, then $\mu$ is $K$-regular.

Proof: Suppose that $\left\{\mu_{n}: n \in \mathbb{N}\right\}$ is not uniformly $K$-regular. Then there exists $U \in \mathcal{N}(G)$ and $a_{0} \in L$ such that for all $b \in K, b \leqslant a_{0}$,

$$
\left\{\mu_{n}(c): c \leqslant a_{0} \ominus b\right\} \not \subset U
$$


for some $n \in \mathbb{N}$. We shall show that, for each $b \leqslant a_{0},(4.1)$ is satisfied for an infinite number of $n \in \mathbb{N}$. If this is not true, there exist $b_{0} \in K, b_{0} \leqslant a_{0}$, and $n_{0} \in \mathbb{N}$ such that $\left\{\mu_{n}(c): c \leqslant a_{0} \ominus b\right\} \subset U$ for all $n>n_{0}$. Since $\mu_{1}, \mu_{2}, \ldots, \mu_{n_{0}}$ are $K$-regular, there are $b_{1}, b_{2}, \ldots, b_{n_{0}} \in K$ such that $b_{j} \leqslant a_{0}$ and $\left\{\mu_{j}(c): c \leqslant a_{0} \ominus b_{j}\right\} \subset U$ for $j=1, \ldots, n_{0}$. Since $K \subset C(L)$ and $K$ is a paving in $L$, there exists $b=\bigvee_{j=0}^{n_{0}} b_{j}$ in $L$ and $b \in K$. Clearly $b \leqslant a_{0}$. Take $c \in L$ such that $c \leqslant a_{0} \ominus b$. Then $c \leqslant a_{0} \ominus b_{j}$ for all $j \in\left\{0,1, \ldots, n_{0}\right\}$, which implies that $\mu_{n}(c) \in U$ for all $n \in \mathbb{N}$, a contradiction.

Therefore, for every $b \in K, b \leqslant a_{0}$, and every $n_{0} \in \mathbb{N}$, there exist $c \in L, c \leqslant a_{0} \ominus b$, and $n>n_{0}$ such that $\mu_{n}(c) \notin U$.

Now, we consider $V \in \mathcal{N}(G)$ such that $V+V \subset U$. Take $b_{0} \in K, b_{0} \leqslant a_{0}$, and $n_{0}=0$. Then there exist $n_{1}>0$ and $c_{1} \leqslant a_{0} \ominus b_{0}$ such that $\mu_{n_{1}}\left(c_{1}\right) \notin U$. Since $\mu_{1}$ is $K$-regular, there exists $b_{1} \in K, b_{1} \leqslant c_{1}$, such that $\mu_{n_{1}}(c) \in V$ for all $c \leqslant c_{1} \ominus b_{1}$. In particular, $\mu_{n_{1}}\left(c_{1}\right)-\mu_{n_{1}}\left(b_{1}\right) \in V$ and so, $\mu_{n_{1}}\left(b_{1}\right) \notin V$. Taking $b_{1} \in K$ and $n_{1} \in \mathbb{N}$, there exist $n_{2}>n_{1}$ and $c_{2} \leqslant a_{0} \Theta b_{1}$ such that $\mu_{n_{2}}\left(c_{2}\right) \notin U$. Following in this way, we can find sequences $\left(b_{k}\right)_{k \in \mathbb{N}}$ in $K$ and $\left(n_{k}\right)_{k \in \mathbb{N}}$ in $\mathbb{N}$ such that $n_{k+1}>n_{k}$, $b_{k+1} \leqslant a_{0} \ominus\left(b_{1} \vee b_{2} \vee \cdots \vee b_{k}\right)$ and $\mu_{n_{k}}\left(b_{k}\right) \notin V$ for all $k \in \mathbb{N}$.

By the construction of $b_{k}$, and using that $K \subset C(L)$, we get that $b_{1} \perp b_{2}$, $b_{1} \vee b_{2}=b_{1} \oplus b_{2}, b_{3} \perp\left(b_{1} \oplus b_{2}\right), \ldots, b_{1} \vee b_{2} \vee \cdots \vee b_{k}=b_{1} \oplus b_{2} \oplus \cdots \oplus b_{k}$ and $b_{k+1} \perp\left(b_{1} \oplus b_{2} \oplus \cdots \oplus b_{k}\right)$. Then, $\left(b_{k}\right)_{k \in \mathbb{N}}$ is an orthogonal sequence in $L$ such that $\mu_{n_{k}}\left(b_{k}\right) \notin V$ for all $k \in \mathbb{N}$. Therefore, $\left\{\mu_{n}: n \in \mathbb{N}\right\}$ is not uniformly $s$-bounded, which contradicts the Brooks-Jewett Theorem 4.1.

REMARKS 4.9 .

(1) An analog of the last theorem can be proved where it is supposed that $L$ is an orthoalgebra and $K$ is a paving in $L$, not necessarily contained in $C(L)$.

(2) Note that if $L$ is a Boolean algebra, then $C(L)=L$ and the condition $K \subset C(L)$ is trivially satisfied.

(3) It is not usual to find, in the literature, a convergence theorem for regular measures. In [32] there is one, which is generalised by our Theorem 4.8 .

\section{CONCLUDing REMARKS}

In the preceding section we have obtained a number of convergence theorems for measures defined on effect algebras. If the effect algebra $L$ is an orthoalgebra (see Example 2.4), then every orthogonal sequence is contained in a Boolean subalgebra of $L$ (see [23]). As a consequence, in order to proof a convergence theorem for measures defined on orthoalgebras, or orthomodular posets, or lattices, it is possible to use the classical results for measures on Boolean algebras (see [3], [4] or [24]). However, this 
method is not valid if $L$ is not an orthoalgebra. For instance, if we consider the effect algebra $L=([0,1], \perp, \oplus, 0,1)$ of Example 2.9 and $A=\{0,1 / 4,1 / 2\} \subset[0,1]$, then $A$ is an orthogonal subset of $[0,1]$, but there is no Boolean subalgebra of $L$ containing $A$ because $1 / 4 \perp 1 / 4$ in $L$ and this is not possible in a Boolean algebra. This argument shows that the effect algebra $L$ does not verify the property WSIP defined by Habil [24]. But $L$ is complete. Hence the property WSIP, as defined in [24], is not weaker than completeness, $\sigma$-completeness or quasi- $\sigma$-completeness in the setting of effect algebras.

Therefore we have proved our convergence theorems by using, not the classical results, but similar technics, when possible, to those used to prove the classical results. The proof of Lemma 3.5, which is the key lemma for the main results given in Section 4, follows basically the ideas of Landers and Rogge [32], adapted to our context: an arbitrary orthogonal set and a sequence of non-commutative measures defined on a quasi- $\sigma$-complete effect algebra. Later, from the Brooks-Jewett theorem, we deduced several results by the following argument: a sequence of measures which are completely additive (respectively $\tau$-smooth, $K$-regular) and uniformly $s$-bounded necessarily is uniformly completely additive (respectively $\tau$-smooth, $K$-regular).

There are still two classical convergence theorems that do not appear in the above section: Nikodým boundedness and Vitali-Hahn-Saks theorems. The first one concerns bounded measures. But, if we bear in mind that there are different ways of defining boundedness for a topological group (see [37] or [46]) and that we are working with non-commutative measures, it turns out that this theorem fails in a certain context (see [4] or [24] for a counterexample), while holding under other conditions, as we can see in $[34]$ or $[37]$.

Finally, we consider the Vitali-Hahn-Saks theorem, that is, the convergence theorem for $\lambda$-continuous measures. We wish to point out that it is easy to find recent results about this theorem for classical measures (see [26] or [47]), while there are very few related works in the setting of non-commutative theory. This happens because the theorem fails, even in the special case of real measures defined on a complete orthomodular lattice, as d'Andrea, de Lucia and Morales have shown in [4] (see also [12]). They construct measures $\mu_{n}, \lambda \in \mathrm{a}\left(L\left(\mathbb{R}^{2}\right), \mathbb{R}\right)$ (see Example 2.7) such that every $\mu_{n}$ is $\lambda$-continuous, $\lim _{n \rightarrow \infty} \mu_{n}(a)=0$ for all $a \in L\left(\mathbb{R}^{2}\right)$ and $\left\{\mu_{n}: n \in \mathbb{N}\right\}$ is not uniformly $\lambda$-continuous. It is easy to see that these measures are uniformly $s$-bounded, uniformly countably (and completely) additive and uniformly $\tau$-smooth. This example also shows that a set of $\lambda$-continuous and uniformly $s$-bounded measures need not to be uniformly $\lambda$-continuous. Thus, a result like [33, Lemma 4.3] or [45, Theorem 2.1] is not true for non-commutative measures.

On the other hand, Dvurečenskij asserts in [12] that if $\mu_{n}, \lambda: L(H) \longrightarrow \mathbb{R}$ are completely additive measures and $H$ is a Hilbert space with dimension bigger or equal 
than 3, then the Vitali-Hahn-Saks theorem holds, even if we change $L(H)$ by $P(\mathcal{A})$, being $\mathcal{A}$ a von Neumann algebra without direct summand of type $I_{2}$ (see [44]).

We can notice that these conditions are exactly those under which the Gleason theorem is valid (see [6] or [36]). From this connection between both theorems, we can ask if the Vitali-Hahn-Saks theorem holds for measures defined on $P(\mathcal{A})$ and taking its values in a Banach space or even in a topological group. But the proof of that result will be based undoubtedly on the Gleason theorem and this is not the subject of this paper.

\section{REFERENCES}

[1] J. Aarnes, 'The Vitali-Hahn-Saks theorem for von Neumann algebras', Math. Scand. 18 (1966), 87-92.

[2] A.D. Alexandroff, 'Additive set functions in abstract spaces II', Mat. Sbornik 9 (1941), 563-628.

[3] A.B. d'Andrea and P. de Lucia, 'The Brooks-Jewett theorem on an orthomodular lattice', J. Math. Anal. Appl. 154 (1991), 507-522.

[4] A.B. d'Andrea, P. de Lucia and P. Morales, 'The Lebesgue decomposition theorem and the Nikodým convergence theorem on an orthomodular poset', Atti Sem. Mat. Fis. Univ. Modena 39 (1991), 73-94.

[5] J.K. Brooks and R.S. Jewett, 'On finitely additive vector measures', Proc. Nat. Acad. Sci. U.S.A. 67 (1970), 1294-1298.

[6] L.J. Bunce and J.D.M. Wright, 'The Mackey-Gleason problem for vector measures on projections in von Neumann algebras', J. London Math. Soc. (2) 49 (1994), 133-149.

[7] D.W. Cohen, An introduction to Hilbert space and quantum logic (Springer-Verlag, Berlin, Heidelberg, New York, 1989).

[8] T.A. Cook, 'The Nikodym-Hahn-Vitali-Saks theorem for states on a quantum logic', in Mathematical foundations of quantum theory, (A.R. Marlow, Editor) (Academic Press, London, 1978), pp. 275-286.

[9] R.B. Darst, 'On a theorem of Nikodým with applications to weak convergence and von Neumann algebras', Pacific J. Math. 23 (1967), 473-477.

[10] J. Diestel and J.J. Uhl, Jr., Vector measures (American Mathematical Society, Providence, RI, 1977).

[11] N. Dunford and J.T. Schwartz, Linear operators, part I: general theory (John Wiley and Sons, New York, 1988).

[12] A. Dvurečenskij, Gleason's theorem and its applications (Kluwer Academic Publishers, Dordrecht, 1993).

[13] A. Dvurečenskij, 'Tensor product of difference posets', Trans. Amer. Math. Soc. 347 (1995), 1043-1057.

[14] A. Dvurečenskij, S. Pulmannová and K. Svozil, 'Partition logics, orthoalgebras and automata', Helv. Phys. Acta 68 (1995), 407-428. 
[15] D. Feldman and A. Wilce, ' $\sigma$-additivity in manuals and orthoalgebras', Order 10 (1993), 383-392.

[16] D.J. Foulis and M.K. Bennet, 'Effect algebras and unsharp quantum logics', Found. Phys. 24 (1994), 1331-1352.

[17] D.J. Foulis, R.J. Greechie and G.T. Rüttimann, 'Filters and supports in orthoalgebras', Internat. J. Theoret. Phys. 31 (1992), 789-807.

[18] F.J. Freniche, 'The Vitali-Hahn-Saks theorem for Boolean algebras with the subsequential interpolation property', Proc. Amer. Math. Soc. 92 (1984), 362-366.

[19] A.M. Gleason, 'Measures on the closed subspaces of a Hilbert space', J. Math. Mech. 6 (1957), 885-893.

[20] R.J. Greechie and D.J. Foulis, 'Transition to effect algebras', Internat. J. Theoret. Phys. 34 (1995), 1369-1382.

[21] R.J. Greechie, D.J. Foulis and S. Pulmannová, 'The center of an effect algebra', Order 12 (1995), 91-106.

[22] S. Gudder, 'Semi-orthoposets', Internat. J. Theoret. Phys. 35 (1996), 1141-1173.

[23] E.D. Habil, 'Orthosummable orthoalgebras', Internat. J. Theoret. Phys. 33 (1994), 1957-1984.

[24] E.D. Habil, 'Brooks-Jewett and Nikodým convergence theorems for orthoalgebras that have the weak subsequential interpolation property', Internat. J. Theoret. Phys. 34 (1995), 465-491.

[25] J. Harding, 'Decompositions in quantum logics', Trans. Amer. Math. Soc. 348 (1996), 1839-1862.

[26] O. Hernández-Lerma and J.B. Lasserre, 'An extension of the Vitali-Hahn-Saks theorem', Proc. Amer. Math. Soc. 124 (1996), 3673-3676.

[27] P.J. Higgins, Introduction to topological groups (Cambridge University Press, Cambridge, 1974).

[28] R.V. Kadison and J.R. Ringrose, Fundamental of the theory of operator algebras, Volumes 1, 2 (Academic Press, London, 1983, 1986).

[29] G. Kalmbach, Orthomodular lattices (Academic Press, London, 1983).

[30] F. Kôpka and F. Chovanec, 'D-posets', Math. Slovaca 44 (1994), 21-34.

[31] P. Kruszyński, 'Probability measures on operator algebras', Reports Math. Phys. 7 (1975), 395-401.

[32] D. Landers and L. Rogge, 'The Hahn-Vitali-Saks and the uniform boundedness theorem in topological groups', Manuscripta Math. 4 (1971), 351-376.

[33] P. de Lucia and P. Morales, 'Some consequences of the Brooks-Jewett theorem for additive uniform semigroup-valued functions', Conference Sem. Math. Univ. Bari 227 (1988).

[34] P. de Lucia and E. Pap, 'Noncommutative version of Nikodým boundedness theorem for uniform space-valued functions', Internat. J. Theoret. Phys. 34 (1995), 981-993.

[35] G.W. Mackey, The mathematical foundations of quantum mechanics (Benjamin Cummings, New York, 1963).

[36] S. Maeda, 'Probability measures on projections in von Neumann algebras', Reviews Math. 
Phys. 1 (1990), 235-290.

[37] J.L. de María and P. Morales, 'A non-commutative version of the Nikodým boundedness theorem.', Atti Sem. Mat. Fis. Univ. Modena 42 (1994), 505-517.

[38] P. Morales, 'A non-commutative version of the Brooks-Jewett theorem', in Proceedings of the first winter school on measure theory, Liptovsky Ján (Slovak. Acad. Sci., Bratislava, 1988), pp. 88-92.

[39] P. Morales and F. García Mazario, 'The support of a measure in ordered topological groups', Atti Sem. Mat. Fis. Univ. Modena 45 (1997), 179-221.

[40] P. Morales, F. García Mazario and P. Jiménez Guerra, 'First Alexandroff decomposition theorem for topological lattice group valued measures', Order 17 (2000), 43-60.

[41] P. Ptáck and S. Pulmannová, Orthomodular structures as quantum logics (Kluwer Academic Publishers, Dordrecht, 1991).

[42] W. Schachermayer, On some classical measure-theoretic theorems for non-sigma-complete Boolean algebras 214, Dissertationes Math. (Rozprawy Mat., Warszawa, 1982).

[43] C. Swartz, An introduction to functional analysis (Marcel Dekker, New York, 1992).

[44] M. Takesaki, Theory of operator algebras I (Springer-Verlag, New York, 1992).

[45] T. Traynor, ' $s$-bounded additive set functions', in Vector and operator valued measures and applications, (D.H. Tucker and H.B. Maynard, Editors) (Academic Press, New York, 1973), pp. 355-365.

[46] H. Weber, 'Compactness in spaces of group-valued contents, the Vitali-Hahn-Saks theorem and Nikodým's boundedness theorem', Rocky Mountain J. Math. 16 (1986), 253-275.

[47] X-D. Zhang, 'On weak compactness in spaces of measures', J. Functional Analysis 143 (1997), 1-9.

Departamento de Matemática Aplicada (E. U. Informática)

Universidad Politécnica de Madrid

28031-Madrid

Spain

e-mail: gmazario@eui.upm.es 